\title{
Pharmacological effects of arecoline on zebrafish behavior, neurochemistry, neurophysiology and brain gene expression
}

\author{
Tamara G. Amstislavskaya \\ Scientific Research Institute of \\ Physiology and Basic Medicine \\ Novosibirsk, Russia \\ amstislavskayatg@physiol.ru \\ DongMei Wang \\ School of Pharmacy, Southwest \\ University, Chongqing, China \\ avkalueff@gmail.com
}

\author{
Nazar Serikuly \\ School of Pharmacy, Southwest \\ University, Chongqing, China \\ avkalueff@gmail.com \\ JingTao Wang \\ School of Pharmacy, Southwest \\ University, Chongqing, China \\ avkalueff@gmail.com
}

Erik T. Alpyshov

School of Pharmacy, Southwest

University, Chongqing, China

avkalueff@gmail.com

Allan V. Kalueff

School of Pharmacy, Southwest

University, Chongqing, China

avkalueff@gmail.com

\begin{abstract}
Arecoline is a naturally occurring psychoactive alkaloid with partial agonism at nicotinic and muscarinic acetylcholine receptors. Arecoline consumption is widespread, making it the fourth (after alcohol, nicotine and caffeine) most used substance by humans. However, the mechanisms of acute and chronic action of arecoline remain poorly understood. Animal models are a valuable tool for CNS disease modeling and drug screening. Complementing rodent studies, the zebrafish (Danio rerio) emerges as a promising novel model organism for neuroscience research. In this study, we assessed the effects of acute and chronic arecoline on adult zebrafish behavior and neurophysiology. Acute and chronic arecoline treatments produce overt anxiolytic-like behavior without affecting general locomotor activity and whole-body cortisol levels. Acute arecoline at $10 \mathrm{mg} / \mathrm{L}$ disrupted shoaling, increased social preference, elevated brain norepinephrine and serotonin levels and reduced serotonin turnover. As it also upregulated early protooncogenes c-fos and c-jun, chronic arecoline increased brain expression of microglial biomarker genes egr-2 and ym1,
\end{abstract}

implicating microglial neuroimmune mechanisms in potential effects of long-term arecoline use. These findings suggest that novel anxiolytic drugs can eventually be developed based on arecoline-like molecules, whose integrative mechanisms of CNS action may involve monoaminergic and neuro-immune modulation.

Keywords - zebrafish; arecoline; CNS; anxiety; genomic effects.

\section{ACKNOWLEDGMENT}

The study was supported by Russian Science Foundation grant 19-15-00053 and budgetary funding for basic scientific research of the Scientific Research Institute of Physiology and Basic Medicine (theme No. AAAA-A16116021010228-0). 